\title{
Day Times Microgram Per Milliliter Per Milligram Per Gram
}

National Cancer Institute

\section{Source}

National Cancer Institute. Day Times Microgram Per Milliliter Per Milligram Per Gram.

NCI Thesaurus. Code C117912.

Day times microgram per milliliter, divided by milligram per gram. 